\title{
Evolution of endoscopic extraperitoneal radical prostatectomy (EERPE): technique and outcome
}

\author{
Jens-Uwe Stolzenburg ${ }^{1}$, Odysseas Andrikopoulos ${ }^{2}$, Panagiotis Kallidonis ${ }^{2}$, Iason Kyriazis ${ }^{2}$, Minh Do ${ }^{1}$ \\ and Evangelos Liatsikos ${ }^{2}$
}

\begin{abstract}
Endoscopic extraperitoneal radical prostatectomy (EERPE) is a well-established and standardized technique for treating patients with localized prostate cancer. Nevertheless, the procedure is continuously being refined with the expansion of anatomical knowledge. The development of a nerve-sparing approach and improvements in currently used equipment are expected to yield better results in cosmesis and convalescence without sacrificing the procedure's established benefits in terms of potency, continence and oncological management. In this study, the technique and its evolution are presented in detail, along with an analysis of its clinical efficacy. We also consult the literature to compare EERPE to transperitoneal laparoscopic radical prostatectomy, and we also discuss new technical advancements regarding the use of robotic assistance during EERPE.
\end{abstract}

Asian Journal of Andrology (2012) 14, 278-284; doi:10.1038/aja.2011.53; published online 19 December 2011

Keywords: extraperitoneal; laparoscopy; prostate cancer; radical prostatectomy; robotic assistance

\section{INTRODUCTION}

Since Raboy et al. ${ }^{1}$ performed the first extraperitoneal endoscopic retropubic radical prostatectomy, endoscopic extraperitoneal radical prostatectomy (EERPE) has evolved into a standardized, replicable procedure for the radical treatment of localized prostate cancer. Over the years, the EERPE technique has been refined to include a nerve-sparing approach, ${ }^{2-6}$ enabling better postoperative continence and potency outcomes. Although the EERPE technique is a minimally invasive surgery, it does not sacrifice clinical and oncological outcomes $^{7}$ for better convalescence and cosmesis. Large clinical trials and ongoing evaluations of the technique are being conducted in demanding patient groups such as obese patients and patients with a history of previous pelvic surgery. These studies show that EERPE is an effective and safe procedure that may become the standard surgical treatment for prostate cancer.

\section{OPERATIVE TECHNIQUE}

Patient positioning and anaesthetic considerations

The procedure is performed under general anaesthesia. An absolute contraindication is severe cardiac insufficiency, while relative contraindications are moderate cardiac insufficiency and chronic pulmonary obstructive disease. Muscle relaxation is used only if required by the surgeon or if lung compliance deteriorates substantially. A balanced anaesthesia technique is used, which also allows the anaesthesiologist to monitor the expiratory gases more efficiently. ${ }^{2,8}$

The laparoscopic tower is placed at the bottom of the operating table. In the original procedure, the patient was positioned in a head-down supine position at $25^{\circ}$, but it was subsequently shown that a head-down supine patient position at $10^{\circ}$ offers better visibility because the bowel does not interfere with the procedure. In addition, the patient's respiratory process is maintained, and possible complications for the anaesthesiologist are reduced. The patient's legs are placed in a straddled position, thus enabling the monitor to be placed almost between them and providing the surgeon with better visibility and comfort. ${ }^{2-6,8,9}$ The patient's hands are placed near the body. The surgeon is positioned on the left side of the patient, the assistant on the right side and the nurse behind the head of the patient to hold the camera because the instruments used in the procedure are limited.

Original operative technique

Preparation of the preperitoneal space and trocar placement. A $12-\mathrm{mm}$ incision is made in the infraumbilical crease lateral to the midline, the anterior rectus fascia is horizontally incised, and the fibres of the rectus muscleare are vertically separated to uncover the posterior rectus fascia. The space between the posterior rectus fascia and the rectus muscle is bluntly dissected, with the fingers pointed towards the preperitoneal space. When the preperitoneal space beneath the arculate line of Douglas is reached, a 12-mm balloon trocar with a $10-\mathrm{mm}$ optical channel is inserted tangentially to the cutaneous plane towards the pubis. The balloon is slowly inflated under direct supervision to ensure that the correct plane (between the rectus muscle and peritoneum) is achieved. The inferior epigastric vessels can be identified ventrally as a point of reference. Once the preperitoneal space has been created, the balloon is desufflated and removed. Vicryl stay sutures size 2-0 are placed in the anterior rectus fascia, and a 10/12-mm Blunt Tip Hassan Trocar is placed in the preperitoneal space. The optics used for the procedure (zero degree optical system) are also placed here. High-flow carbon

${ }^{1}$ Department of Urology, University of Leipzig, Leipzig D-04103, Germany and ²Department of Urology, University of Patras, Rio, Patras 26500, Greece Correspondence: Dr P Kallidonis (pkallidonis@yahoo.com)

Received: 5 December 2010; Revised: 24 March 2011; Accepted: 26 April 2011; Published online: 19 December 2011 
dioxide insufflation is initiated and maintained at a pressure of $12 \mathrm{mmHg}$ to prevent subcutaneous emphysema.

After the establishment of pneumoperitoneum, a 5-mm trocar is placed through the rectus muscle sheath between the iliac spine and the umbilicus on the left side of the abdominal wall. Under direct vision, two 5-mm trocars are placed in the contralateral side under the hypogastrium. The first trocar is placed cranially in the right pararectal line. After insertion of these two trocars, dissection to the contralateral side is performed with care to avoid tearing the peritoneum (especially in patients with previous surgical history, such as an appendectomy). When the peritoneum has been adequately dissected, a $12-\mathrm{mm}$ trocar is placed approximately two fingers' breadth medial to the left anterior superior iliac spine. The lateral left $12-\mathrm{mm}$ trocar is used to introduce the needles and remove the lymph node packages and the endobag containing the specimen. In extremely tall and obese patients, the trocars must be placed $1-3 \mathrm{~cm}$ caudally for better access to the preperitoneal space.

The dissection of the preperitoneal space is completed by identifying the following structures (from outside to inside): the spermatic cord, the external iliac vessels, the pubic arc with Cooper's ligament, the symphysis, and finally the bladder and the prostate. Any inguinal and/or incidental hernias present, must be dissected and retracted to facilitate subsequent lymph node extraction and prostatectomy. At this point, the pelvic lymph nodes can be dissected. ${ }^{10}$

Exposure of anatomic landmarks and ligation of the Santorini Plexus. Exposure of the prostate and the bladder neck followed by incision of the endopelvic fascia is the next step in the procedure. Care must be taken not to injure the Santorini plexus. Incision on both sides of the endopelvic fascia is sufficient to expose the levator ani muscle. During this step of the procedure, the assistant must retract the prostate medially to free any fibres of the levator ani muscle still attached to the prostate. In addition, any venous vessels lateral to the puboprostatic ligaments should be dissected to ensure good haemostasis. The same holds true for the puboprostatic ligaments. The Santorini plexus is then ligated using Vicryl sutures; a straightened UR-6 2-0 needle is appropriate for the ligation. The dorsal venous complex is not divided until prostate dissection is complete.

Dissection of the bladder neck. After identification of the rim between the mobile bladder neck and the solid prostate, using the catheter, a transversal incision is made from the 11:00 to 1:00 o'clock position at the bladder neck, and the urethra is developed with a sharp and blunt dissection. The urethra is then incised, and the deflated balloon catheter becomes visible. Then, the catheter is elevated and fixed by the assistant in the direction of the symphysis into the retropubic space, and the dissection continues in the lateral direction. At this point, the assistant elevates the ventral part of the prostate in a ventral direction to identify the natural groove between the bladder mucosa and prostate in the dorsal direction. The posterior bladder neck is then transected by sharp dissection. To identify the ureteral orifices in the case of a large prostatic middle lobe, the use of double-J catheters may be helpful but is not necessary.

Mobilisation of seminal vesicles and the prostate and dissection of the urethra. The anterior portion of Denonvillier's fascia is perforated horizontally in the midline and dissected until the ampullary portions of the vasa deferentia and the seminal vesicles become visible. The arteries supplying the seminal vesicles are coagulated and sectioned with monopolar scissors.

After the seminal vesicles have been completely mobilized, the assistant elevates them towards the symphysis, which provides excellent visibility for the incision and dissection of the posterior layer of Denonviller's fascia. To avoid rectal injury, the dissection must be as close to the prostate as possible while being directed towards the apex of the prostate. Using the Ultracision device (Ethicon Endo-Surgery Inc., Cincinatti, $\mathrm{OH}, \mathrm{USA}$ ) to avoid bleeding, both prostate pedicles are systematically coagulated and divided close to the prostate. This dissection is performed on both sides, and complete anterior, lateral and posterior prostate mobilisation is achieved. Then, the Santorini plexus is sectioned, and the urethra is carefully dissected. It is important to preserve as much of the external sphincter as possible; thus, care is taken to avoid coagulation of the urethral wall. The external sphincter and the urethra are sharply divided from the apex of the prostate in a tangential direction, and the cutting line is extended ventrocranially to caudodorsally until the catheter becomes visible. Its tip is then lifted by the assistant to expose the lateral and dorsal limit of the prostatic apex and the urethra, and the dissection is completed in a vertical direction.

Removal of the specimen. In $20 \%$ of cases, the prostate is removed immediately via an endobag. In patients with larger prostates, removal is delayed until the end of the operation: the trocar in the left iliac fossa is removed, and the incision is enlarged by 3 or $4 \mathrm{~cm}$. After prostate removal, the fascia is sutured to avoid $\mathrm{CO}_{2}$ leakage, and the trocar is again placed into the preperitoneal space.

Urethrovesical anastomosis. Following these manoeuvres, urethrovesical anastomosis is conducted using seven to nine interruptible sutures (a running suture can be used as an alternative) in the following order. The first three are performed posteriorly at the 8:00, 6:00 and 4:00 o'clock positions, and a catheter is then inserted to guide the subsequent sutures. The lateral portion is completed in the same manner (two sutures at the 9:00 and 3:00 o'clock positions), and the two final stitches are placed in the 1:00 and 11:00 o'clock positions. All seven sutures are performed with the same "outside-inside" (bladder) and "inside-outside" (urethra) technique, thus allowing waterproof sealing of the anastomosis and also ensuring the extraluminal tying of the sutures. The bladder is then filled with $150 \mathrm{ml}$ sterile water via the catheter to evaluate the presence of extravasation through the anastomosis.

Finally, a 20 Charriere Robinson drain (Boehringer Ingelheim, FRG, Germany) is placed into the retropubic space through the 5$\mathrm{mm}$ port site in the right iliac fossa and kept there for 24-48 h. After removal of the trocars, the skin is conventionally sutured, except for the wounds from the two $12-\mathrm{mm}$ trocars that require suturing of the fascia to prevent herniation. ${ }^{9}$

\section{The nerve-sparing approach}

Increased familiarity with the procedure and improved knowledge of periprostatic anatomy have enabled the development of a nerve-sparing approach. ${ }^{2,3,5,10}$ Before the preparation and dissection of the dorsal bladder neck, it is important to incise the fascia overlaying the anterior aspect of the prostate and to mobilize both neurovascular bundles. Complete dissection of the bladder neck can then be achieved. To avoid any intraprostatic preparation, the dissection must be carefully performed in the correct plane. After this step, the anatomical landmarks of the ampullae and seminal vesicles are visualized, 
and the procedure continues as previously described (complete bladder neck-sparing technique). While dissecting the seminal vesicles, their tips can be left in place, and extra care must be taken to avoid injuring the neurovascular bundles that are in close proximity to the seminal vesicles. ${ }^{2}$

The incision or blunt dissection of the thin fascia over the anteriorlateral aspect of the prostate during dorsal bladder neck dissection enables mobilisation and subsequent preparation of the neurovascular bundles (NVBs) (anterolaterally near the apex and dorsally near the base). The prostate is retracted to the left or right, accordingly. For better visibility, the posterior layer of Denonviller's fascia is completely divided. The prostatic pedicles are then dissected, and the NVBs are completely separated from the prostate using an Ultracision or Sonosurge (Olympus, Winter and IBE, Hamburg, Germany) device. Importantly, we found that the use of either of these devices resulted in a poorer functional outcome compared with using clips and cold scissors to dissect the pedicles. ${ }^{11}$ The energy produced by the ultrasonic devices results in injury to the NVBs and pedicles. Using clips and cold scissors is now the standard procedure for NVB and prostatic pedicle preparation during nerve-sparing EERPE at our institution.

\section{Development of the intrafascial nerve-sparing EERPE-further evolution of the technique}

The intrafascial nerve-sparing modification to the procedure was the next step in the evolution of EERPE. A better understanding of periprostatic anatomy, the establishment of nomenclature for the structures surrounding the prostate (especially the neurovascular bundles and the periprostatic fascia) and consequently an appreciation of the level of dissection required for sufficient nerve preservation have led to significant advancements in the technique.

Several study groups ${ }^{11-14}$ have shown particular interest in elucidating the aforementioned relations, but a lack of conclusive evidence has failed to provide a unanimously accepted finding, Using their experience with robotic-assisted surgeries Menon et al. ${ }^{12}$ subsequently reported that the neurovascular bundles transversed the periprostatic fascia. Their conclusion was further supported by including in their technique an anterior incision of the periprostatic fascia in a manner parallel to the neurovascular bundles. Costello et al. ${ }^{13}$ suggested that the largest portion of the neurovascular bundles descend posteriorly to the seminal vesicle, and that the nerves converge to the midprostatic level only to diverge once again near the apex. Consequently, the authors proposed a wide dissection of the lateral aspect of the prostate. In a more recent study, Kiyoshima et al. ${ }^{14}$ found the neurovascular bundles to be located on the postero-lateral regions of the prostate in $52 \%$ of patients. In the remaining $48 \%$ of patients, the bundles were spread throughout the entire lateral aspect of the prostate. Walsh ${ }^{15}$ suggested that the neurovascular bundle is located between the two layers of the lateral pelvic fascia, and they posit that if nerve preservation is desired, the prostatic fascia must remain on the prostate.

These conclusions, especially those proposed by Menon et al., demonstrated the necessity and catalysed the development of the intrafascial nerve-sparing EERPE. ${ }^{4,6}$ In this technique, placement of the patient and the trocars are identical to the previous technique. However, the first step is dissection of the bladder neck, which starts at the 12:00 o'clock position and continues until the longitudinal musculature of the bladder neck is completely revealed. ${ }^{6}$ Incision of the bladder neck is then extended from 10:00 to 2:00 o'clock. With the bladder neck as the starting point, an incision of the periprostatic fascia is made bilaterally and directed distally towards the apex while remaining medial to the puboprostatic ligaments. In this stage, a detachment of the prostate's 'envelopment' is possible. If all the manoeuvres are performed correctly to maintain the lateral aspects of the periprostatic fascia, the endopelvic fascia and the puboprostatic ligaments are separated intact. Then, the lateral plane between the bladder neck and the prostate is developed, with care taken to not involve the attached lateral tissues. The dorsal bladder neck is then carefully dissected without injuring the NVBs. Seminal vesicle preparation is also performed with caution, and Denonviller's fascia is not incised but bluntly dissected strictly in the midline. Thus, the planes for dissecting the prostatic pedicles are created, as the prostate is now held in place only by the prostatic pedicles and the apex. The prostatic pedicles are clipped and cut in a stepwise fashion. The remaining pedicles attached to the prostate fascias are dissected. Finally, the Santorini plexus is ligated, the urethra is dissected and the vesicourethral anastomosis is performed.

\section{COMPLICATIONS}

EERPE has been established in specialized urological centres, and the technique has been refined over time with the accumulation of surgical experience. Despite these advancements, complications are always present. ${ }^{16,17}$ Recent reviews have estimated the incidence of EERPErelated complications to range from $2 \%$ to $17 \% .{ }^{16,18,19}$ Because these complications are directly related to the level of experience of the surgeon, it is important for them to be promptly recognized and efficiently treated, if not prevented.

Injury to the inferior epigastric vessels is one of the most common intraoperative complications, and it usually occurs during insertion of the fourth trocar (pararectal line, right iliac fossa). This complication can be avoided by using the laparoscope to carefully inspect the abdominal wall before trocar insertion or by inserting a fine needle to designate the course of the trocar prior to insertion. Other methods for preventing this injury include assistance with the suction tube thus exerting pressure from the inner surface of the trocar insertion point. If vessel injury does occur, the use of bipolar coagulation and clipping is recommended. If the bleeding is persistent, suturing with the aid of a straight needle through the abdominal wall to ligate the bleeding vessel is recommended. In all cases, the area must be carefully inspected after partial $\mathrm{CO}_{2}$ deflation to avoid injuries hidden by a temporary tamponade from the direct pressure of the gas. Additionally, inserting the laparoscope through the left $12-\mathrm{mm}$ trocar while the right trocar is removed always helps to identify vascular injuries.

Another common site of vascular injury is the Santorini plexus, and this injury can be avoided by careful ligation. If injury does occur, the treatment depends on the persistence of the bleeding. An initial increase of insufflation pressure to $20 \mathrm{mmHg}$ is necessary, followed by bipolar coagulation and additional sutures, with care to avoid damaging the adjacent structures. In the case of persistent bleeding, haemostasis is achieved through the complete dissection of the ventral urethral wall and retraction of the catheter to facilitate tamponade for 5-10 min. Injury of the iliac vein is possible and is most commonly caused by abrupt trocar insertion. This complication can be managed by laparoscopic suturing or a conversion to open laparotomy.

Any patient experiencing persistent abdominal pain within 2 weeks following EERPE must be carefully examined for bowel injuries (perforation or thermal injury). Other nonspecific symptoms may include vomiting, malaise, distension, faecaluria and the presence of bubbles within the urine. In addition, fever and leucocytosis may be present, and septic shock can develop. The placement of the lateral trocars presents a theoretical risk for a potential bowel injury if the lateral attachments of the peritoneum are not carefully mobilized. Incision of 
the peritoneum provides better visibility of the trocars and may be necessary in some cases. However, the most common cause of bowel injury is the dissection of the apex dorsally near the end of the procedure. Previous studies have shown that bowel injury can be detected by insufflating gas into the rectum and filling the operative field with water (when lacerations are present, bubbles can be seen) along with the use of intrarectal devices such as the rectal bougie balloon. ${ }^{20}$ However, the latter device does not completely prevent the occurrence of rectal injuries. If the injury is identified intraoperatively, endoscopic correction with a two-layer suture line is necessary, followed by parenteral nutrition for 3 days and residual-free enteral feeding for a minimum of 6 days. If the injury is not found intraoperatively, open surgical correction is necessary. Khoder et al. ${ }^{21}$ have reported successful conservative management in rare cases, but further studies are necessary to confirm the efficacy of such practices.

Transection of lymphatic vessels often leads to lymphoceles. This can be avoided by using a combination of bipolar coagulation, harmonic scalpels and clipping. If it does occur, lymphoceles can be treated with fenestration of the peritoneum, percutaneous drainage or sclerotherapy. The incidence of lymphoceles is significantly higher for the extraperitoneal approach. Fenestration of the peritoneum during EERPE and lymphadenectomy significantly reduces the incidence of lymphoceles. ${ }^{22} \mathrm{~A}$ patient with a lymphocele may present with pelvic pain, voiding problems following catheter removal, painful leg edema or even hydronephrosis, depending on the size and site of the lymphocele. Fever is a common symptom of infected lymphoceles.

Injuries to the bladder and ureters are possible complications of this procedure. The former occur mostly during the dissection of the extraperitoneal space and in patients with a history of extraperitoneal hernioplasty with mesh placement. ${ }^{16}$ An infusion of 200 -ml saline into the bladder can identify this complication, and it is repaired in a single layer. Ureter injuries can occur during an extensive lymphadenectomy or during posterior bladder neck dissection and anastomosis. In both cases, the injury can be prevented by injecting indigo carmine or by catheterising both the ureters to visualize the orifices. A lesion can be detected by administrating indigo carmine with furosemide and watching for dye leakage.

Hernia formation is an infrequent occurrence, mostly appearing after specimen removal. Careful suturing of the external oblique fascia prevents this complication. ${ }^{16}$

Leakage through the anastomosis is not uncommon. Intraoperatively, the instillation of saline into the bladder through the urethral catheter is useful for detecting extravasation sites. The solution used depends on the size and location of the leakage. Additional suturing and/or placement of a mono J catheter may be sufficient to treat small leaks that are mostly anterolateral, but a complete revision of the anastomosis is sometimes necessary for larger leaks. Tension applied through prolonged catheterisation may also be sufficient to manage minor leakages.

The catheter should be removed on the fifth postoperative day following retrograde cystoscopy or 3-7 days later in the case of urine extravasation. Removing the catheter earlier than the fifth day is directly associated with acute urinary retention due to perioperative edema. ${ }^{23}$ Guillonneau et al. ${ }^{23}$ showed that the risk of retention following catheter removal on the first peri-operative day is $100 \%$ and is reduced to $3.2 \%$ by day 4 . In our series, this complication was successfully treated in all cases by catheterisation for an additional 1-4 days. ${ }^{16}$ Other less common complications include obturator nerve damage, catheter blockage and gas embolism.

\section{CLINICAL EVALUATION}

To date, the largest set of studies concerning the clinical outcomes of EERPE are those of Stolzenburg et al..$^{9,22,24-28}$ In the largest and most recent of these studies, the clinical results of 2400 patients are presented. ${ }^{28}$ The indications for using EERPE were the same as for open and laparoscopic radical prostatectomy. Obese patients and patients with previous a pelvic surgery were not excluded. Prostate-specific antigen (PSA) $>10 \mathrm{ng} \mathrm{ml}^{-1}$ and/or the presence of a tumour at stage G3 or with a Gleason score above 6 were the indications for pelvic lymph node dissection. The bilateral nerve-sparing procedure was performed in preoperatively potent patients with $\mathrm{T} 1$ or $\mathrm{T} 2$ disease, $\mathrm{PSA}<10 \mathrm{ng} \mathrm{ml}^{-1}$ and a Gleason score $\leqslant 3+4$. A unilateral nervesparing procedure was conducted in patients with a Gleason score of $4+3$ or $4+4$ or with a palpable tumour (as analysed by frozen sectioning contralaterally to the tumour side. A small group of patients with a high Gleason score of $4+3$ or PSA $>10 \mathrm{ng} \mathrm{ml}^{-1}$ requested and underwent the bilateral nerve-sparing procedure.

The perioperative data were as follows: the mean patient age was 63.3 years, the mean preoperative PSA level was $9.8 \mathrm{ng} \mathrm{ml}^{-1}$ and the mean operating time was $150.7 \mathrm{~min}$. The mean blood loss was $255 \mathrm{ml}$, while the transfusion rate was $0.7 \%$. Conversion to open prostatectomy was never deemed necessary. Pelvic lymph node dissection was conducted in $50.8 \%(n=1219)$ of patients, extending to the obturator fossa and external iliac vessels (initially), while a more extensive lymphadenectomy to the level of the common iliac vessels was performed in the majority of the cases. Bilateral endoscopic extraperitoneal radical prostatectomy (nsEERPE) was performed in 28.0\% (672/2400) of the patients and unilateral nsEERPE in $11.8 \%$ (284/2400) of the patients. One hundred patients underwent intraoperative hernia repair with mesh placement. Histological results showed that $70.4 \%$ $(1693 / 2400)$ of the patients had $\mathrm{pT}_{2}$ stage disease, and $8.0 \%(136 /$ $2400)$ of these individuals had positive surgical margins; $29.5 \%$ $(699(136 / 2400))$ of the patients had $\mathrm{pT}_{3}$ disease, and $35.6 \%$ (249/ 2400) of these had positive surgical margins. Metastasis to the lymph nodes was observed in $6.1 \%$ (75/2400) of the patients who underwent pelvic lymph node dissection.

Overall continence was observed in $71.7 \%, 81.4 \%$ and $94.7 \%$ of the cases at 3, 6 and 12 months, respectively. Continence rates in the patients who underwent bilateral nsEERPE were 79.5\%, $87.3 \%$ and $96.6 \%$ at the same time points, respectively. Postoperative potency results in 956 patients treated with nsEERPE showed a favourable outcome for the bilateral group during the follow-up period. Younger patients, especially those $<55$ years old, tended to have better postoperative potency results ( $84.9 \%$ at 12 months postoperation).

The total complication rate was $0.41 \%$ intraoperatively, $9.44 \%$ in the early postoperative period and $0.28 \%$ in the late postoperative period. Rectal injuries occurred in eight cases and were successfully treated with two-layer suture management. Interventional management during the first month following EERPE was required in 85 of the 2400 patients (3.54\%). Late complications, including four anastomotic strictures, were treated with additional interventions in a total of seven cases $(0.29 \%)$. Anastomotic leakage was found in 63 patients $(2.6 \%)$ and was treated by prolonged catheterisation.

Liatsikos et al. ${ }^{29}$ presented the clinical outcome of EERPE in relation to obesity by retrospectively evaluating 500 cases. The patients were categorized into three groups according to body mass index (BMI) (WHO classification): BMI $<25.0 \mathrm{~kg} \mathrm{~m}^{-2}$ (normal weight), BMI between 25 and $29 \mathrm{~kg} \mathrm{~m}^{-2}$ (overweight) and BMI $>30.0 \mathrm{~kg} \mathrm{~m}^{-2}$ (obese). Age, prostate size and preoperative PSA level were similar in all three groups. The mean BMI was $27 \mathrm{~kg} \mathrm{~m}^{-2}$ for the cohort, 
and $26.8 \%, 56.6 \%$ and $16.6 \%$ of the patients comprised the normal, overweight and obese groups, respectively. Pelvic lymph node dissection and nsEERPE were performed in 218 and 123 patients, respectively. Obese patients showed a significantly higher American Society of Anesthesiologists score. The mean blood loss during the operation was $200 \mathrm{ml}$. No conversions to open surgery or blood transfusion were necessary, and the mean operative time was $149 \mathrm{~min}$. There was a statistically significant difference in operation duration among the groups, as EERPE or nsEERPE required an additional $20 \mathrm{~min}$ of operating time for obese patients. At 3 months postoperation, obese patients showed a nonsignificant trend towards poorer continence results, but this was not found at 6 months postoperation. The duration of catheterisation and the transfusion rate were not significantly different among the groups.

EERPE is considered technically more demanding in patients who have previously undergone transurethral resection of the prostate. Do et $a l^{30}$ described the clinical outcomes of 100 of these cases, 26 of which underwent nsEERPE. Operative time and mean blood loss were similar to the standard EERPE population, while the transfusion rate and mean duration of catheterisation were slightly higher. Positive surgical margin rates were $7 \%$ for pT2 and $36 \%$ for $\mathrm{pT} 3 / 4$ stage disease. In total, $93 \%$ of the patients were continent, and $94 \%$ of the patients showed PSA $\leqslant 0.1 \mathrm{ng} \mathrm{ml}^{-1}$ at 12 months postoperatively. The overall complication rate was $14 \%$. Potency rates for the nsEERPE group were $52.6 \%$ and $66.7 \%$ at 6 and 12 months, respectively.

Other sets of studies on EERPE and its technique variations have also been published. Tobias-Machado et al. ${ }^{31}$ described the results of 28 cases who underwent a procedure virtually identical to the open technique. The mean surgical time for this cohort was 280 min, mean blood loss was $320 \mathrm{ml}$ and median hospital stay was 3 days. Two rectal lesions occurred as intraoperative complications, and three cases of extraperitoneal urinary fistula occurred as postoperative complications. Of 18 previously potent patients, five presented with erectile dysfunction.

Poulakis et al. ${ }^{32}$ presented a prospective comparison between the single-knot running and interrupted suturing techniques during vesicourethral anastomosis in 250 patients. The population was divided into two groups of 125 patients, and the outcomes of the two techniques were compared. Recorded parameters were similar for both techniques, except for the mean operative time (16 vs. $24 \mathrm{~min}$ ) and the difficulty score, which both favoured the single-knot technique. The continence rates at 3 and 6 months postoperation were $76 \%$ and $91.5 \%$, respectively, for Group 1 and $77.6 \%$ and 93\%, respectively, for Group 2. Complication rates did not significantly differ between the groups.

Srinualnad et al. ${ }^{33}$ described their findings in 27 EERPE cases and compared them to 55 cases of open prostatectomy performed by the same surgical group. Mean operative times were $268 \mathrm{~min}$ (EERPE group) and $157 \mathrm{~min}$ (open group) $(P<0.01)$. Median blood losses were 500 and $1000 \mathrm{ml}$ for the EERPE and open groups, respectively $(P<0.001)$. The transfusion rate was higher in the open group than the EERPE group, while hospitalisation time and pathological stage did not differ between the two groups. In the EERPE group, there were two rectal complications. Apart from operative time, EERPE proved to be safer and more feasible compared with the open technique, without sacrificing oncological outcome.

Blana et al. ${ }^{34}$ reported the impact of previous laparoscopic prostatectomy experience on the surgeon's ability to master EERPE. The technique was performed on 120 consecutive patients by two surgeons with different experiences, and their learning curves were analysed and compared. Operation time was the only parameter that significantly differed between the two surgeons, whereas complication rates and early oncological results were similar. McNeill et al..$^{35}$ evaluated the clinical data and the surgeon's learning curve from 300 consecutive patients who underwent EERPE. The estimated probability of a complication decreased from $29 \%$ for the first procedure to $<1 \%$ for the two hundred and fiftieth procedure. In addition, the data showed a decrease in operative duration and positive surgical margins rates as the surgeon gained more experience. The continence rate and biochemical recurrence-free rate were $89 \%$ and $94 \%$, respectively, for the first 100 patients at a 12-month follow-up visit. These results showed that EERPE can be replicated after a mentored surgical fellowship training, and that it has a continuous learning curve.

As surgical techniques continually evolve, the concept of scarless surgery comes closer to reality. Rabenalt et al. ${ }^{36}$ described their first experience with single-site EERPE in a 74-year-old patient. The Triport device was utilized, and both straight and pre-curved instruments were used to duplicate the standard EERPE technique. No complications occurred intraoperatively or postoperatively, and surgical margins were negative.

Studies have also compared EERPE to the transperitoneal approach, and these reports have particularly focused on the comparison between EERPE and transperitoneal laparoscopic radical prostatectomy (TLRP) $)^{37-50}$ in terms of operative time, complications, oncological results, hospitalisation duration and convalescence (results shown also on Table 1). Most studies agree that EERPE requires less

Table 1 Comparison of the main perioperative and functional parameters in the extraperitoneal and transperitoneal approaches

\begin{tabular}{|c|c|c|c|c|c|c|c|c|c|}
\hline \multirow[t]{2}{*}{ Study } & \multicolumn{2}{|c|}{$\begin{array}{c}\text { Mean operative time } \\
\text { (min) }\end{array}$} & \multicolumn{2}{|c|}{ Mean blood loss (ml) } & \multicolumn{2}{|c|}{$\begin{array}{c}\text { Potency rates at } 12 \\
\text { months (\%) }\end{array}$} & \multicolumn{2}{|c|}{$\begin{array}{c}\text { Continence rates at } 12 \\
\text { months (\%) }\end{array}$} & \multirow[t]{2}{*}{$\begin{array}{l}\text { Positive surgical } \\
\text { margins (\%) }\end{array}$} \\
\hline & $T L R P$ & EERPE & TLRP & EERPE & $T L R P$ & EERPE & TLRP & EERPE & \\
\hline Eden et al. $(2004)^{38}$ & 239 & 191 & 310 & 201 & 61 & 82 & 90 & 96 & - \\
\hline Erdogru et al. $(2004)^{39}$ & 197 & 212 & - & - & - & - & 86 & 84 & NSD \\
\hline Ruiz et al. $(2004)^{40}$ & 248 & 220 & 678 & 803 & - & - & - & - & NSD (23 vs. 29.7) \\
\hline Remzi et al. $(2005)^{43}$ & 279 & 217 & 290 & 189 & - & - & 85 & 88 & - \\
\hline Phinthusophon et al. (2007) ${ }^{50}$ & 350 & 220 & 883 & 605 & - & - & - & - & NSD \\
\hline
\end{tabular}

Abbreviations: EERPE, endoscopic extraperitoneal radical prostatectomy; NSD, no statistical differences; TLRP, transperitoneal laparoscopic radical prostatectomy. -, not available. 
Table 2 Main perioperative parameters and preliminary results of robotic-assisted EERPE

\begin{tabular}{|c|c|c|c|c|c|c|}
\hline Study group & No. of patients & $\begin{array}{l}\text { Mean operative time } \\
\text { (min) }\end{array}$ & $\begin{array}{l}\text { Mean blood loss } \\
(\mathrm{ml})\end{array}$ & Continence rates (\%) & Potency rates (\%) & $\begin{array}{l}\text { Positive surgical } \\
\text { margins (\%) }\end{array}$ \\
\hline Atug and Thomas $(2007)^{47}$ & 40 & 229 & 221 & - & - & 20 \\
\hline Joseph et al. $(2006)^{53}$ & 325 & 130 & 196 & - & - & 13 \\
\hline Madi et al. $(2007)^{49}$ & 55 & 214 & 125 & - & - & 23.5 \\
\hline Ploussard et al. (2009) ${ }^{54}$ & 175 & 140 & 350 & - & - & 17.2 (in pT2) \\
\hline Chung et al. $(2010)^{52}$ & 155 & 150 & 350 & 98.6 (12 months) & - & 10.5 (in pT2) \\
\hline Xylinas et al. (2010) ${ }^{57}$ & 50 & 127 & 335 & 60 (1 month) & 66 (1 month) & 12 \\
\hline
\end{tabular}

Abbreviations: EERPE, endoscopic extraperitoneal radical prostatectomy. —, not available; pT2: tumor confined to the prostate.

operative time and has an easier learning curve compared to TLRP, but it must be noted that many groups have reported that the two approaches did not differ in these aspects. These studies proposed that each surgeon should decide which procedure to use according to personal preference and experience. ${ }^{37,44}$ However, other studies have demonstrated the superiority of EERPE over TLRP in terms of patient history (obesity, inguinal hernia and previous pelvic surgery), ${ }^{39}$ intraand perioperative complications ${ }^{42}$ and overall morbidity. ${ }^{43}$

If a robot-assisted approach to EERPE is selected, ${ }^{51}$ it is necessary for the surgeon to have experience in the transperitoneal approach before performing EERPE because of the lack of operating space in the procedure. ${ }^{47}$ Nevertheless, the equally favourable outcome of roboticassisted EERPE in experienced hands has been proven, ${ }^{44}$ as shown in the comparative studies by Porpiglia et al. ${ }^{46}$ and Atug et al. ${ }^{47}$ Recent studies by Madi et al., ${ }^{49}$ Chung et al. ${ }^{52}$ Joseph et al. ${ }^{53}$ and Ploussard et $a l .{ }^{54}$ have verified that the extraperitoneal approach offers the same perioperative results as the intraperitoneal approach. In fact, the extraperitoneal approach is superior in terms of postoperative pain and major complications (e.g., bowel injury). The results of these studies are presented in Table 2.

Stolzenburg et al. ${ }^{55,56}$ evaluated the impact of previous surgical history on the performance and outcome of EERPE. In a total of 500 patients, no statistical significance was detected between the 'no surgery' and 'previous surgery' groups in terms of overall operative time, positive surgical margin status and complications or reinterventions. However, the authors noted that EERPE was subjectively more technically demanding in the case of mesh placement for patients with a previous hernia surgery. Nevertheless, this fact did not interfere with overall patient outcome and is not considered a contraindication.

\section{CONCLUSION}

The current literature suggests that EERPE has the potential to become the standard treatment for localized prostate cancer. Its feasibility and safety extend to difficult and demanding patient groups (e.g., obese patients or patients having undergone previous pelvic surgery), ${ }^{55,56}$ and the development of an intrafascial and nerve-sparing approach has resulted in improved continence and decreased instances of erectile function without sacrificing oncological outcome.

\section{COMPETING FINANCIAL INTERESTS}

The authors declare no competing financial interests

1 Raboy A, Ferzli G, Albert P. Initial experience with extraperitoneal endoscopic radical retropubic prostatectomy. Urology 1997; 50: 849-53.

2 Stolzenburg JU, Truss MC, Do M, Rabenalt R, Pfeiffer $\mathrm{H}$ et al. Evolution of endoscopic extraperitoneal radical prostatectomy (EERPE) — technical improvements and development of a nerve-sparing, potency-preserving approach. World J Urol 2003; 21: 147-52
3 Stolzenburg JU, Rabenalt R, Do M, Tannapfel A, Truss MC et al. Nerve-sparing endoscopic extraperitoneal radical prostatectomy: University of Leipzig technique. J Endourol 2006; 20: 925-9.

4 Stolzenburg JU, Rabenalt R, Tannapfel A, Liatsikos E. Intrafascial nerve-sparing endoscopic extraperitoneal radical prostatectomy. Urology 2006; 67: 17-21.

5 Stolzenburg JU, McNeill A, Liatsikos EN. Nerve-sparing endoscopic extraperitoneal radical prostatectomy. BJU Int 2008; 101: 909-28.

6 Stolzenburg JU, Rabenalt R, Do M, Schwalenberg T, Winkler M et al. Intrafascial nerve-sparing endoscopic extraperitoneal radical prostatectomy. Eur Urol 2008; 53: 931-40.

7 Paul A, Ploussard G, Nicolaiew N, Xylinas E, Gillion N et al. Oncologic outcome after extraperitoneal laparoscopic radical prostatectomy: midterm follow-up of 1115 procedures. Eur Urol 2010; 57: 267-72.

8 Stolzenburg JU, Aedtner B, Olthoff D, Koenig F, Rabenalt R et al. Anaesthetic considerations for endoscopic extraperitoneal and laparoscopic transperitoneal radical prostatectomy. BJU Int 2006; 98: 508-13.

9 Stolzenburg JU, Do M, Pfeiffer H, König F, Aedtner B et al. The endoscopic extraperitoneal radical prostatectomy (EERPE): technique and initial experience. World J Urol 2002; 20: 48-55.

10 Stolzenburg JU, Liatsikos EN, Rabenalt R, Do M, Sakelaropoulos G et al. Nerve sparing endoscopic extraperitoneal radical prostatectomy - effect of puboprostatic ligament preservation on early continence and positive margins. Eur Urol 2006; 49: 103-11.

11 Walsh PC. Anatomic radical prostatectomy: evolution of the surgical technique. J Urol 1998; 160: 2418-24.

12 Menon M, Tewari A, Peabody J. Vattikuti Institute prostatectomy: technique. J Urol 2003; 169: 2289-92.

13 Costello AJ, Brooks M, Cole OJ. Anatomical studies of the neurovascular bundle and cavernosal nerves. BJU Int 2004; 94: 1071-6.

14 Kiyoshima K, Yokomizo A, Yoshida T, Tomita K, Yonemasu H et al. Anatomical features of periprostatic tissue and its surroundings: a histological analysis of 79 radical retropubic prostatectomy specimens. Jpn J Clin Oncol 2004; 34: 463-8.

15 Walsh PC. Anatomical studies of the neurovascular bundle and cavernosal nerves. J Urol 2005; 174: 566.

16 Stolzenburg JU, Rabenalt R, Do M, Lee B, Truss MC et al. Complications of endoscopic extraperitoneal radical prostatectomy (EERPE): prevention and management. World $\mathrm{J}$ Urol 2006; 24: 668-75.

17 Stolzenburg JU, Rabenalt R, Do M, Lee B, Truss MC et al. Categorisation of complications of endoscopic extraperitoneal and laparoscopic transperitoneal radical prostatectomy. World J Urol 2006; 24: 88-93.

18 Gillitzer R, Melchior SW, Hampel C, Wiesner C, Fichtner J et al. Specific complications of radical perineal prostatectomy: a single institution study of more than 600 cases. J Urol 2004; 172: 124-8.

19 Catalona WJ, Carvalhal GF, Mager DE, Smith DS. Potency, continence and complication rates in 1,870 consecutive radical retropubic prostatectomies. J Urol 1999; 162: 433-8.

20 Rassweiler J, Sentker L, Seemann O, Hatzinger M, Stock C et al. Heilbronn laparoscopic radical prostatectomy. Technique and results after 100 cases. Eur Urol 2001; 40: 54-64.

21 Khoder WY, Becker AJ, Schlenker B, Tritschler S, Bastian PJ et al. Conservative management of rectal perforation after nerve sparing endoscopic extraperitoneal radical prostatectomy (nSEERPE) in a patient with a past history of polypectomy. Eur J Med Res 2009; 14: 320-2.

22 Stolzenburg JU, Do M, Rabenalt R, Pfeiffer H, Horn L et al. Endoscopic extraperitoneal radical prostatectomy: initial experience after 70 procedures. J Urol 2003; 169: 2066-71.

23 Guillonneau B, Rozet F, Cathelineau X, Lay F, Barret E et al. Perioperative complications of laparoscopic radical prostatectomy: the Montsouris 3-year experience. J Urol 2002; 167: 51-6.

24 Stolzenburg JU, Truss MC, Rabenalt R, Do M, Pfeiffer $\mathrm{H}$ et al. Endoscopic extraperitoneal radical prostatectomy. Results after 300 procedures. Urologe $A$ 2004; 43: 698-707. in German.

25 Stolzenburg JU, Rabenalt R, DO M, Ho K, Dorschner W et al. Endoscopic extraperitoneal radical prostatectomy: oncological and functional results after 700 procedures. J Urol 2005; 174: 1271-5.

26 Stolzenburg JU, Rabenalt R, Do M, Truss MC, Burchardt M et al. Endoscopic extraperitoneal radical prostatectomy: the University of Leipzig experience of 1,300 cases. World J Urol 2007; 25: 45-51. 
27 Stolzenburg JU, Rabenalt R, Do M, Kallidonis P, Liatsikos EN. Endoscopic extraperitoneal radical prostatectomy: the University of Leipzig experience of 2000 cases. J Endourol 2008; 22: 2319-25.

28 Stolzenburg JU, Kallidonis P, Minh D, Dietel A, Häfner T et al. Endoscopic extraperitoneal radical prostatectomy: evolution of the technique and experience with 2400 cases. J Endourol 2009; 23: 1467-72.

29 Liatsikos E, Mühlstädt S, Kallidonis P, Rabenalt R, Do M et al. Performance and functional outcome of endoscopic extraperitoneal radical prostatectomy in relation to obesity: an assessment of 500 patients. BJU Int 2008; 102: 718-22.

30 Do M, Haefner T, Liatsikos E, Kallidonis P, Hicks J et al. Endoscopic Extraperitoneal radical prostatectomy after previous transurethral resection of prostate: oncologic and functional outcomes of 100 cases. Urology 2010; 75: 1348-52.

31 Tobias-Machado M, Lasmar MT, Medina JJ, Forseto PH Jr, Juliano RV et al. Preliminary experience with extraperitoneal endoscopic radical prostatectomy through duplication of the open technique. Int Braz J Urol 2005; 31: 228-35.

32 Poulakis V, Skriapas K, de Vries R, Dillenburg W, Witzsch U et al. Vesicourethral anastomosis during endoscopic extraperitoneal radical prostatectomy: a prospective comparison between the single-knot running and interrupted technique. Urology 2006; 68: 1284-9.

33 Srinualnad S, Nualyong C, Udompunturak S, Kongsuwan W. Endoscopic extraperitoneal radical prostatectomy (EERPE): a new approach for treatment of localized prostate cancer. J Med Assoc Thai 2006; 89: 1601-8.

34 Blana A, Straub M, Wild PJ, Lunz JC, Bach T et al. Approach to endoscopic extraperitoneal radical prostatectomy (EERPE): the impact of previous laparoscopic experience on the learning curve. BMC Urol2007; 7: 11

35 McNeill AS, Nabi G, McLornan L, Cook J, Bollina P et al. Endoscopic extraperitoneal radical prostatectomy: critical analysis of outcomes and learning curve. BJU Int2010; 106: 1537-43.

36 Rabenalt R, Arsov C, Giessing M, Winter C, Albers P. Extraperitoneal laparoendoscopic single-site radical prostatectomy: first experience. World J Urol 2010; 28: 705-8.

37 Cathelineau X, Cahill D, Widmer H, Rozet F, Baumert H et al. Transperitoneal or extraperitoneal approach for laparoscopic radical prostatectomy: a false debate over a real challenge. J Urol 2004; 171: 714-6.

38 Eden CG, King D, Kooiman GG, Adams TH, Sullivan ME et al. Transperitoneal or extraperitoneal laparoscopic radical prostatectomy: does the approach matter? J Urol 2004; 172: 2218-23.

39 Erdogru T, Teber D, Frede T, Marrero R, Hammady A et al. Comparison of transperitoneal and extraperitoneal laparoscopic radical prostatectomy using match-pair analysis. Eur Urol 2004; 46: 312-9.

40 Ruiz L, Salomon L, Hoznek A, Vordos D, Yiou R et al. Comparison of early oncologic results of laparoscopic radical prostatectomy by extraperitoneal versus transperitoneal approach. Eur Urol 2004; 46: 50-4.

41 Stolzenburg JU, Truss MC, Bekos A, Do M, Rabenalt R et al. Does the extraperitoneal laparoscopic approach improve the outcome of radical prostatectomy? Curr Urol Rep 2004; 5: 115-22.
42 Brown JA, Rodin D, Lee B, Dahl DM. Transperitoneal versus extraperitoneal approach to laparoscopic radical prostatectomy: an assessment of 156 cases. Urology 2005; 65: 320-4.

43 Remzi M, Klingler HC, Tinzl MV, Fong YK, Lodde M et al. Morbidity of laparoscopic extraperitoneal versus transperitoneal radical prostatectomy verus open retropubic radical prostatectomy. Eur Urol 2005; 48: 83-9

44 Atug F, Castle EP, Woods M, Srivastav SK, Thomas R et al. Transperitoneal versus extraperitoneal robotic-assisted radical prostatectomy: is one better than the other? Urology 2006; 68: 1077-81.

45 Gao ZL, Wu JT, Wang K, Wang L, Yang DD et al. Comparison of the extraperitoneal and transperitoneal laparoscopic radical prostatectomy. Chin Med J (Engl) 2006; 119: 2125-8.

46 Porpiglia F, Terrone C, Tarabuzzi R, Billia M, Grande S, et al. Transperitoneal versus extraperitoneal laparoscopic radical prostatectomy: experience of a single center. Urology 2006; 68: 376-80.

47 Atug $\mathrm{F}$, Thomas $\mathrm{R}$. Transperitoneal versus extraperitoneal robotic-assisted radical prostatectomy: which one? Minerva Urol Nefrol 2007; 59: 143-7.

48 Capello SA, Boczko J, Patel HR, Joseph JV. Randomized comparison of extraperitoneal and transperitoneal access for robot-assisted radical prostatectomy. J Endourol 2007; 21: 1199-202.

49 Madi R, Daignault S, Wood DP. Extraperitoneal v intraperitoneal robotic prostatectomy: analysis of operative outcomes. J Endourol 2007; 21: 1553-7.

50 Phinthusophon K, Nualyong C, Srinualnad S, Taweemonkongsap T, Amornvesukij T. Laparoscopic radical prostatectomy: transperitoneal laparoscopic radical prostatectomy versus extraperitoneal endoscopic radical prostatectomy. J Med Assoc Thai 2007; 90: 2644-50.

51 Esposito MP, Ilbeigi P, Ahmed M, Lanteri V. Use of fourth arm in da Vinci robotassisted extraperitoneal laparoscopic prostatectomy: novel technique. Urology 2005; 66: 649-52.

52 Chung JS, Kim WT, Ham WS, Yu HS, Chae Y et al. Comparison of oncological results, functional outcomes, and complications for transperitoneal versus extraperitoneal robot-assisted radical prostatectomy: a single surgeon's experience. J Endourol 2011; 25: 787-92.

53 Joseph JV, Rosenbaum R, Madeb R, Erturk E, Patel HR. Robotic extraperitoneal radical prostatectomy: an alternative approach. J Urol 2006; 175: 945-50.

54 Ploussard G, Xylinas E, Salomon L, Vordos D, Hoznek A et al. Robot-assisted extraperitoneal laparoscopic radical prostatectomy: experience in a high-volume laparoscopy reference centre. BJU Int 2010; 105: 1155-60

55 Stolzenburg JU, Anderson C, Rabenalt R, Do M, Ho K et al. Endoscopic extraperitoneal radical prostatectomy in patients with prostate cancer and previous laparoscopic inguinal mesh placement for hernia repair. World J Urol 2005; 23: 295-9.

56 Stolzenburg JU, Ho KM, Do M, Rabenalt R, Dorschner W et al. Impact of previous surgery on endoscopic extraperitoneal radical prostatectomy. Urology2005; 65: 32531.

57 Xylinas E, Ploussard G, Salomon L, Paul A, Gillion N et al. Intrafascial nerve-sparing radical prostatectomy with a laparoscopic robot-assisted extraperitoneal approach: early oncological and functional results. J Endourol 2010; 24: 577-82. 